\title{
METODOLOGÍA PARA LA MITIGACIÓN DE RIESGOS EN EL MEJORAMIENTO DE VÍAS TERCIARIAS EN CUNDINAMARCA, COLOMBIA
}

\section{Methodology for the mitigation of risks in the improvement of tertiary roads in Cundinamarca, Colombia}

\author{
Juan Sebastián Sánchez Estrada ${ }^{1}$, \\ ${ }^{1}$ Universidad Militar Nueva Granada, Facultad de ingeniería, \\ Programa de Maestría de gerencia de proyectos. \\ Email:u21100156@unimilitar.edu.co
}

(Recibido Agosto 22 de 2020 y aceptado Noviembre 19 de 2020)

\section{Resumen}

El presente documento se basa en la investigación realizada en la gestión de riesgos para proyectos de tipo vial, especialmente, en el mejoramiento de vías terciarias. El cual consiste en el análisis de los riesgos aplicados a este tipo de proyectos, así como, en la presentación de propuestas de mitigación y reducción de la probabilidad de impacto y de ocurrencia. Con este fin, se realizó la identificación de riesgos y análisis propuesto por el Project Management Institute (PMI), para elaborar un manual, el cual propone una metodología y un paso a paso para realizar la correcta construcción del pavimento rígido reduciendo los riesgos latentes a su ocurrencia y los imprevistos laborales en la obra de construcción.

El manual para la gestión de riesgos en el mejoramiento de vías terciarias fue aplicado en un proyecto de obra civil en el municipio de Soacha, Cundinamarca. Este dejó resultados positivos, en donde se observó una alta disminución en los impactos de los riesgos establecidos en principio. Lo anterior, se vio reflejado tanto en la disminución de los riesgos, como en el aumento del rendimiento de la obra civil, así también, hubo una disminución en costos generados por imprevistos, lo cual hace que la utilidad sea mayor que la proyectada inicialmente.

Palabras clave: riesgos, impacto, vía terciaria, placa huella, veredas, imprevistos.

\begin{abstract}
This paper is based on research conducted in risk management for road-type projects, especially, in the improvement of tertiary roads. It consists of the analysis of the risks applied to these types of projects, as well as the presentation of proposals for mitigation and reduction of the probability of impact and occurrence. Based on this, the risk identification and analysis proposed by the Project Management Institute (PMI) was carried out to develop a manual, which proposes a methodology and a step by step to carry out the proper construction of the rigid pavement reducing the risks latent to its occurrence and the work contingencies in the construction workplace.
\end{abstract}

The manual for risk management in the improvement of tertiary roads was applied in a civil works project in the municipality of Soacha, Cundinamarca. This left positive results, where a high decrease in the impacts of risks established in principle was observed. This was reflected both in the decrease in risks and in the increase in the performance of civil works, as well as in the decrease in unforeseen costs, which makes the utility greater than initially was projected.

Key words: risks, impact, tertiary route, footprint plate, sidewalks, contingencies. 


\section{INTRODUCCIÓN}

La ejecución de las vías terciarias en el país genera desarrollo en las zonas en donde se intervienen este tipo de proyectos, pero, al no desarrollarlos de manera adecuada puede crear un conflicto en la comunidad cercana.

Las zonas que necesitan el mejoramiento de la vía terciaria son localidades en vulnerabilidad, en donde su economía depende de varios factores, generalmente, son zonas agrícolas [1] y la población necesita transportar productos a mercados para generar capital.

La investigación se desarrolló con el fin de reducir los imprevistos ocasionados en la construcción de pavimentos por placa huella [2], que mitiguen los impactos de los riesgos presentados en cada una de las actividades en la planeación y ejecución del proyecto.

A través de la metodología del PMI [3] en la gestión de los riesgos de un determinado proyecto, se aplicó a este tipo de obra civil, con el fin de recaudar los riesgos latentes en la construcción de la vía por placa huella, y también conocer la probabilidad de ocurrencia, nivel de impacto y actividades de mitigación del mismo.

En los cuales se logra desarrollar una metodología en la práctica de la construcción del mejoramiento de vías terciarias por Placa huella [4] para que los impactos y riesgos sean minimizados, así mismo, reducir los imprevistos en la obra civil.

Cuando en una obra de construcción se logran reducir los imprevistos, indirectamente, se va a ver reflejado en el presupuesto del proyecto, lo cual ocasiona que las utilidades esperadas por el trabajo van a ser mayores para los contratistas y ejecutores de este tipo de proyectos.

\section{MÉTODOS}

En la búsqueda y análisis de los riesgos que se presentan en la ejecución de proyectos de vías terciarias, la cual se realizó mediante la metodología propuesta por el PMI (Project Management Institute), se encontraron 29 riesgos, los cuales afectan de manera directa e indirecta el alcance, tiempo de ejecución, presupuesto y calidad de la obra.

Se aplicó un análisis en los riesgos presentados en proyectos de características similares, también desarrollados en el departamento de Cundinamarca. De estos se correlacionó entre los demás contratos para saber el número de ocasiones que se presentó dicho riesgo, y de esta forma, tener la probabilidad de ocurrencia.

Por último, se realizó la determinada gestión de riesgos propuesta por el PMI [5], en el cual se llega a la implementación de la metodología sugerida con actividades de mitigación para poder reducir el impacto y ocurrencia de los riesgos.

\section{RESULTADOS}

Los riesgos presentes en este tipo de proyectos, están latentes en varias de sus actividades, los cuales se presenta a continuación:

Tabla 1 Riesgos en el mejoramiento de vía terciaria

\section{No. RIESGO IDENTIFICADO}

R1 Mal estado de las máquinas
R2 Costos elevados de alquiler
R3 Desgaste acelerado en piezas y lubricantes
R4 Robo de maquinaria
R5 Baja capacidad técnica y cognitiva por cuenta
de la mano de obra
R6 No existencia de personal calificada en la zona
de ejecución del proyecto
R7 Mala compactación y desprendimiento de
material
R8 $\begin{aligned} & \text { Altos costos en precio de materiales por alta } \\ & \text { demanda }\end{aligned}$


R9 Baja calidad de materiales

R10 Demoras en despacho de materiales

R11 Poca opción de financiamiento

R12 Opción de financiamiento

R13 Llega a destiempo de insumos para las actividades

R14 Llegada de materiales de manera incompleta

R15 Mal cálculo en cuantías de insumos

R16 Llegada en mal estado del pedido

R17 Corte y figurado de manera inapropiada a los planos, desgaste de herramienta de corte

R18 Alto desperdicio

R19 Mala calidad de material de tablas

R20 Alto desperdicio y mal manejo

R21 Mal estado de la máquina

R22 Daño mecánico de la mezcladora

R23 Escasez de combustible para el funcionamiento

R24 Dosificación de concreto que no sea la correcta

R25 El acero de refuerzo no está ubicado de manera correcta

R26 Mal almacenamiento del material (cemento)

R27 Clima inapropiado para fundir

R28 Fisuras considerables en la placa de concreto

R29 Accidente laboral

Los riesgos presentes en la ejecución del mejoramiento de las vías terciarias, tienen con ellos un nivel de impacto [6], el cual se ve reflejado de manera diferente a las restricciones iniciales del proyecto, como bien lo son el alcance, el tiempo de ejecución, el presupuesto y la calidad de la obra.

Es por lo anterior que, se realizó la gestión en cada una de las restricciones, con el fin de determinar cuál de estas es más susceptible a cada uno de los impactos ocasionados por los riesgos ya mencionados.

La Figura 1, muestra como el alcance del proyecto tiene un impacto mínimo frente a los riesgos presentados, el mayor nivel de impacto, pero con poca probabilidad de ocurrencia es el R8 (Altos costos en precio de materiales por alta demanda).

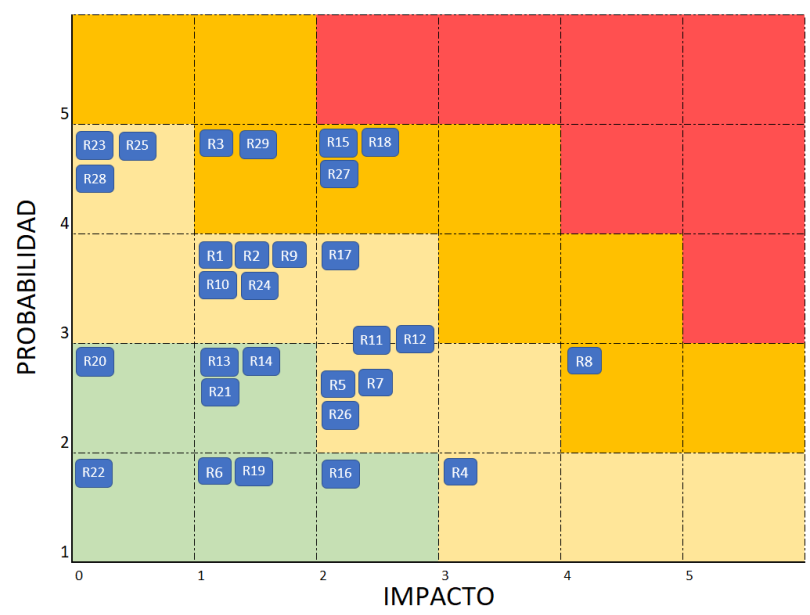

Figura 1. Probabilidad VS Impacto (Alcance)

Para el tiempo de ejecución del proyecto, es afectado de manera alta pero poco probable, aunque cabe resaltar que esta variable debe de ser supervisada y controlada de buena forma, ya que este punto tiene criterios contractuales y legales.

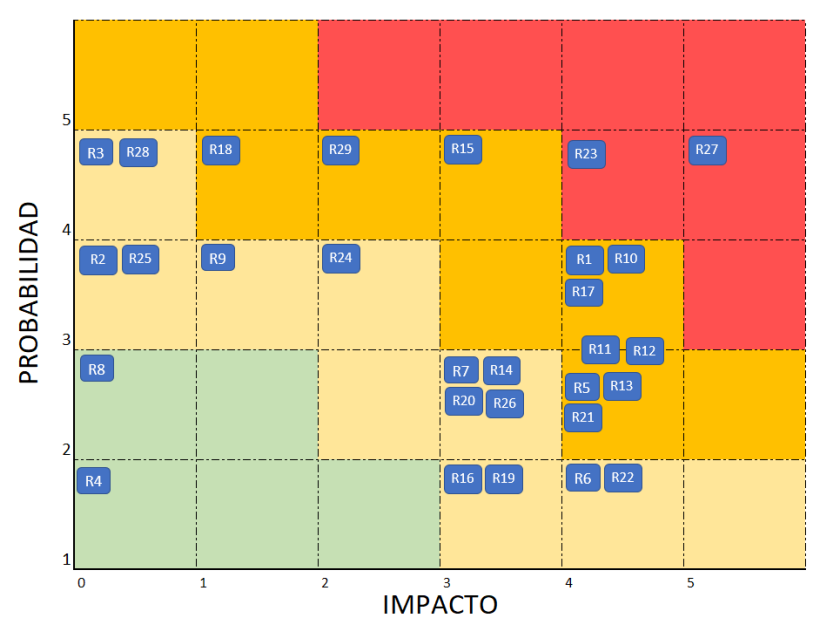

Figura 2. Probabilidad VS Impacto (Tiempo)

La Figura 3, correspondiente al presupuesto se logra evidenciar que los riesgos tienen mayor impacto frente a los costos del presupuesto, es decir, el 
presupuesto es la variable con mayor vulnerabilidad.

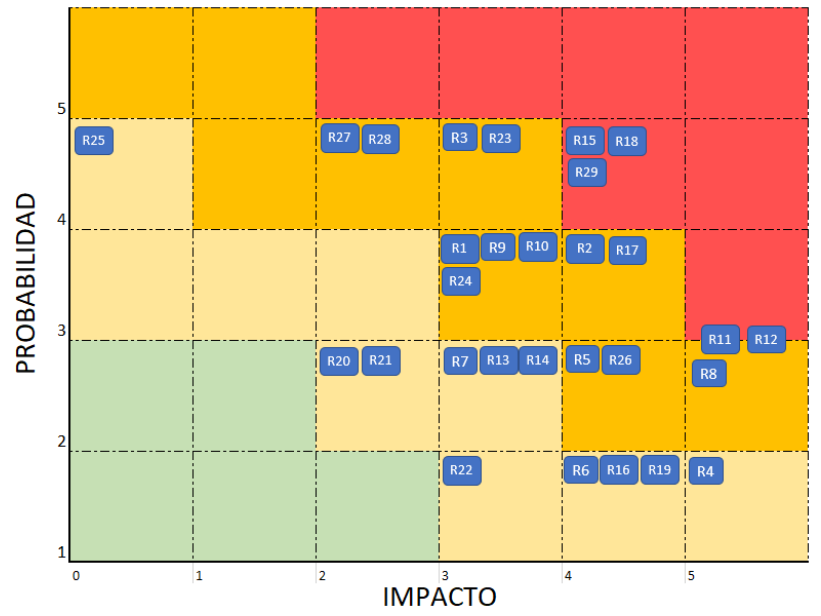

Figura 3. Probabilidad VS Impacto (Presupuesto)

Para la calidad de la obra, la Figura 4, muestra que no muchos riesgos afectan esta restricción, pero los impactos y probabilidades son considerables, los cuales pueden perjudicar el proyecto.

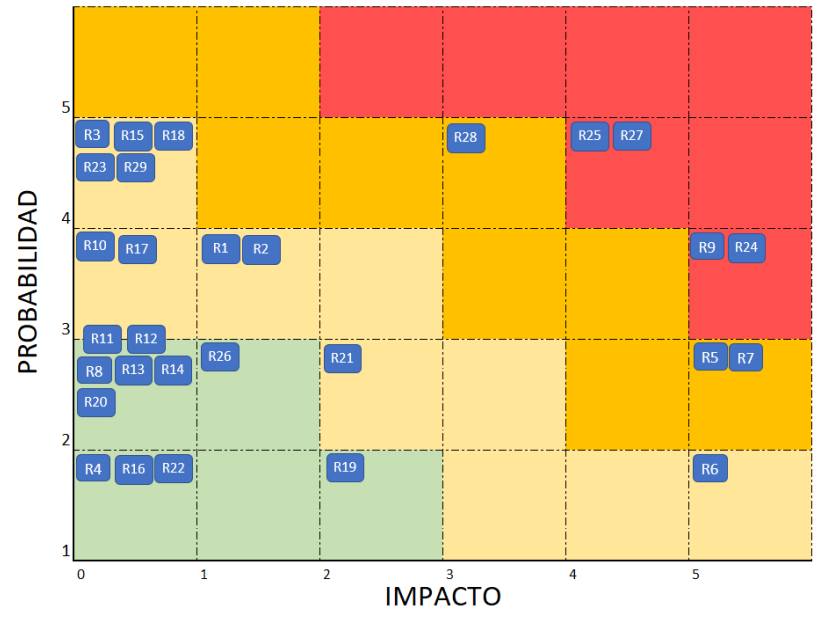

Figura 4. Probabilidad VS Impacto (Calidad)

La calidad y estabilidad de la obra es un tema contractual, el cual tiene responsabilidades para el constructor, es por ello que se deberá realizar la construcción con los mejores estándares de calidad. En este sentido, los impactos de los riesgos deben ser mínimos para que esta restricción no se vea perjudicada.

Posterior a determinar los impactos proporcionados por los riesgos en cada una de las restricciones, y aclarar cuál de estas tiene mayor susceptibilidad en cada una de estas marcas; se procede a determinar medidas preventivas las cuales disminuyan la ocurrencia e impacto de los riesgos.

Con cada una de estas medidas de mitigación, se realizó el "Manual para la Gestión de Riesgos en el Mejoramiento de Vías Terciarias", el cual fue implementado en el municipio de Soacha Cundinamarca, en el contrato de obra pública 2156 de 2019, el cual tiene como objeto el "Mejoramiento de la vía terciaria que conduce de la vereda Hungría a la vereda San Jorge en el sector.

Para este se implementaron las medidas de prevención junto con una metodología constructiva, la cual fue comparada a la hora de su terminación, con un contrato de similitud económica, física y técnica demostrando mejores resultados en cuanto a la disminución de riesgos, disminución de imprevistos y, así mismo, mayor utilidad económica.

\section{DISCUSIÓN}

En la siguiente tabla, se presentan los flujos de caja correspondientes a cada uno de los proyectos, de acuerdo a la programación de ejecución de la obra civil.

Tabla 2. Comparación flujos de caja

\begin{tabular}{ccc}
\hline ZIPAQUIRÁ & SOACHA \\
\hline SEM. & $\begin{array}{c}\text { BCWS } \\
\text { CPTP }\end{array}$ & $\begin{array}{c}\text { BVWS } \\
\text { CPTP }\end{array}$ \\
\hline 1 & $\$ 1.500 .000 .00$ & $\$ 3.750 .000 .00$ \\
2 & $\$ 10.736 .760 .00$ & $\$ 19.338 .872 .00$ \\
3 & $\$ 19.096 .077 .69$ & $\$ 28.085 .040 .50$ \\
4 & $\$ 25.751 .423 .84$ & $\$ 40.097 .901 .57$ \\
5 & $\$ 32.406 .7693 .99$ & $\$ 50.079 .058 .00$ \\
6 & $\$ 36.097 .896 .42$ & $\$ 52.191 .474 .67$ \\
\hline
\end{tabular}


$7 \$ 42.151 .424 .99$

$\$ 68.598 .726 .00$

$8 \$ 48.204 .953 .56$

$9 \$ 57.068 .250 .99$

$10 \$ 59.100 .623 .49$

$11 \$ 61.166 .185 .99$

$12 \$ 68.299 .114 .56$

$13 \$ 87.586 .900 .99$

Los anteriores datos demuestran que la metodología propuesta tiene resultados positivos. El rendimiento aumentó de acuerdo a la metodología propuesta y los costos de inversión son menores, estos se ven reflejados en el mismo final de la obra civil, simplemente, que dos metodologías constructivas diferentes.

En la Gráfica 5, se muestra como el proyecto ejecutado en el municipio de Soacha, tiene mayor cantidad de inversión, el manejo apropiado de las finanzas se ven reflejadas de forma positiva.

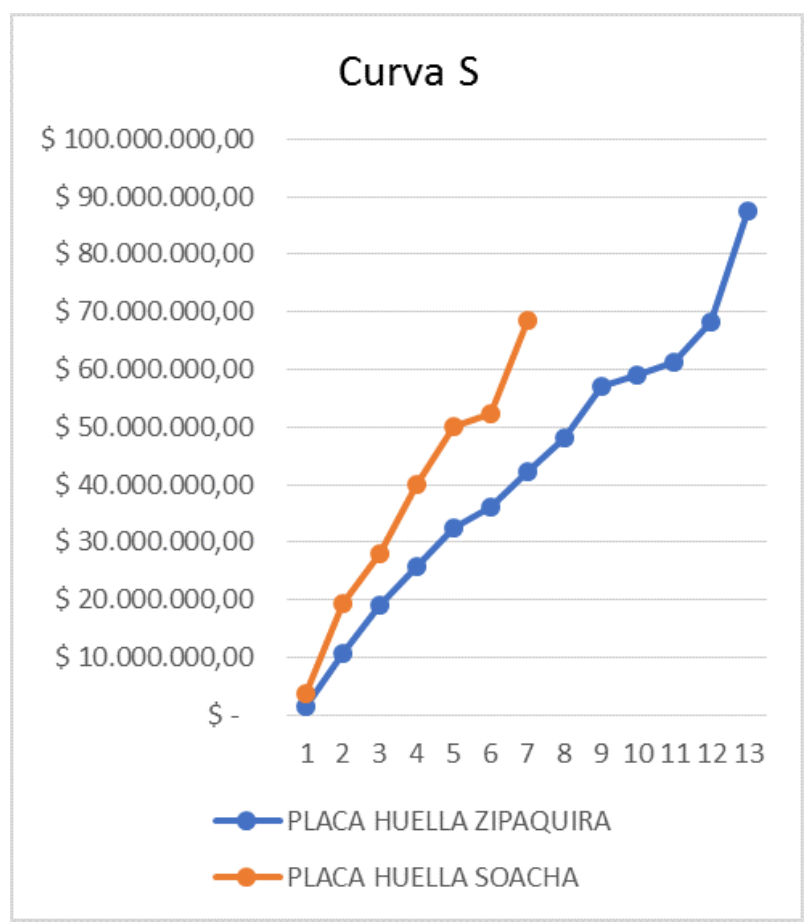

Figura 5. Comparativa curva S

\section{CONCLUSIONES}

La restricción con mayor vulnerabilidad es el presupuesto de la obra, ya que cada una de las otras restricciones se apoyan de manera significativa en esta y la calidad de la obra se debe tener en cuenta para evitar riesgos jurídicos.

En la comparación de los proyectos de mejoramiento de vía terciaria en Soacha y Zipaquirá, Cundinamarca se logra concluir que al aplicar la metodología propuesta se logra aumentar en un aproximado 16\%-19\% las utilidades generadas.

La implementación de la metodología propuesta aumento el rendimiento por metro lineal, es decir, la misma cantidad de obra se ejecutó en un 54\% menos a comparación de la obra ejecutada en el municipio de Zipaquirá, disminuyendo costos del día a día.

Cada uno de los riesgos se presentan de manera diferente en cada uno de los factores mencionados, pero en el que mayor relevancia tiene es en el presupuesto oficial del proyecto, ya que este es vulnerable y susceptible a cambios y modificaciones, poniendo en vilo la utilidad final del contratista.

El presupuesto es el factor más vulnerable en la ejecución de la obra civil, es por ello que se deberá reducir la ocurrencia de los riesgos, ya que en su mayoría tienen un alto impacto en este factor; es por esto que es importante tener en cuenta medidas preventivas para que estos no estén presentes a lo largo del desarrollo del proyecto.

Los riesgos que impactan la calidad del proyecto son riesgos que se pueden mitigar con una amplia actividad de prevención, las cuales hacen que estos riesgos reduzcan su impacto de manera considerable.

\section{REFERENCIAS}

[1] F. Remolina, «PMI Colombia,» 21 Abril 2015. [En línea]. Available: http://www.pmicolombia.org/blog/ 
conceptos-basicos-de-la-gestion-de-riesgos-paralos-miembros-del-equipo-del-proyecto/. [último acceso: 18 Noviembre 2018].

[2] Colombia Compra Eficiente, «Colombia Compra Eficiente,» 1901 2017. [En línea]. Available: https:// www.colombiacompra.gov.co/secop/secop-i. [último acceso: 1911 19].

[3] E. Correa Valderrama, «El rol de las vías terciarias en la construcción de un nuevo pais,» Revista de Ingenieria, no 45, pp. 64-71, 2017.

[4] L. Ameijide García, Gestión de proyectos según el PMI, Barcelona, Cataluña: Universidad Oberta de Catalunya, 2016, p. 70.

[5] INVIAS, Guia de Diseño de Pavimento con Placa-hueIla, vol. 1, Bogota: Ministerio de Transporte, 2017.

[6] INVIAS, Manual de Diseño Geométrico de Carreteras, Bogota, 2008, p. 298.

[7] H. KERZNER, Project Management, A Sistems Approach to Planning, Scheduling, and Controlling, 12th ed., New Jersey: Jhon Wiley \& Sons, 2013.

[8] L. Narváez, "Vías terciarias: Motor del desarrollo económico rural.,» Revista de Ingenieria, no 45, pp. 80-87, Junio 2017. 